\title{
On the Optimality of Single-Carrier Transmission in Large-Scale Antenna Systems
}

Antonios Pitarokoilis, Saif Khan Mohammed and Erik G. Larsson

\section{Linköping University Post Print}

N.B.: When citing this work, cite the original article.

Original Publication:

Antonios Pitarokoilis, Saif Khan Mohammed and Erik G. Larsson, On the Optimality of Single-Carrier Transmission in Large-Scale Antenna Systems, 2012, IEEE Wireless Communications Letters, (1), 4, 276-279.

http://dx.doi.org/10.1109/WCL.2012.041612.120046

Copyright: (C2012 IEEE. Personal use of this material is permitted. However, permission to reprint/republish this material for advertising or promotional purposes or for creating new collective works for resale or redistribution to servers or lists, or to reuse any copyrighted component of this work in other works must be obtained from the IEEE.

Postprint available at: Linköping University Electronic Press http://urn.kb.se/resolve?urn=urn:nbn:se:liu:diva-80173 


\title{
On the Optimality of Single-Carrier Transmission in Large-Scale Antenna Systems
}

\author{
Antonios Pitarokoilis, Saif Khan Mohammed, Erik G. Larsson
}

\begin{abstract}
A single carrier transmission scheme is presented for the frequency selective multi-user (MU) multiple-input singleoutput (MISO) Gaussian Broadcast Channel (GBC) with a base station (BS) having $M$ antennas and $K$ single antenna users. The proposed transmission scheme has low complexity and for $M \gg K$ it is shown to achieve near optimal sum-rate performance at low transmit power to receiver noise power ratio. Additionally, the proposed transmission scheme results in an equalization-free receiver and does not require any MU resource allocation and associated control signaling overhead. Also, the sum-rate achieved by the proposed transmission scheme is shown to be independent of the channel power delay profile (PDP). In terms of power efficiency, the proposed transmission scheme also exhibits an $O(M)$ array power gain. Simulations are used to confirm analytical observations.
\end{abstract}

Index Terms-Single-Carrier Transmission, Large MIMO.

\section{INTRODUCTION}

Multiple-input multiple-output (MIMO) systems have attracted significant research interest during the last decade due to various advantages they promise, both in single user [1] and multiuser channels [2]. It has been recently shown that the employment of an excess of antennas at the BS (very large MIMO) offers unprecedented array and multiplexing gains both in the uplink and in the downlink [3], [4]. The array gain offered by very large MIMO systems allows for power savings that scale as $1 / M$ and $1 / \sqrt{M}$ with perfect and imperfect channel state information (CSI) respectively, where $M$ is the number of BS antennas [5]. The multiplexing gains offered by very large MIMO allows tens of users to be allocated the entire system bandwidth simultaneously. This eliminates to a large extent the need for resource allocation and the associated control signaling overhead. Since each user communicates over the whole system bandwidth, even low per user spectral efficiencies can result in very high per user throughput. In a MU-MISO GBC with $K$ users and $M \gg K$ (very large MIMO), a low per user spectral efficiency implies an operating regime where the ratio of the total transmit power to the receiver additive noise power is small. Since MU interference at each receiver is proportional to the total transmit power, the additive noise dominates over MU interference and therefore even suboptimal precoding algorithms (like beamforming with the conjugate transpose of the channel gain matrix) have near optimal performance.

The authors are with the Department of Electrical Engineering (ISY), Linköping University, 58183 Linköping, Sweden (email: \{antonispit, saif, erik.larsson\}@isy.liu.se). This work was supported by the Swedish Foundation for Strategic Research (SSF) and ELLIIT. E. G. Larsson is a Royal Swedish Academy of Sciences (KVA) Research Fellow supported by a grant from the Knut and Alice Wallenberg Foundation.
Previous results for very large MIMO systems have only considered frequency flat channels [3], [4], [5]. In this paper we consider a MU-MISO frequency selective GBC with $M \gg K$. For this channel OFDM (OFDMA) is an attractive transmission scheme as it facilitates scheduling in the frequency domain and simplifies receiver equalization. However, there is a substantial price to pay for this. OFDM comes at a loss in spectral and power efficiency owing to the insertion of cyclic prefix. Moreover, the signals resulting from OFDM modulation have a very large peak-to-average ratio, requiring the RF power amplifiers to work with a large power backoff and in an operating regime where they have low efficiency. For this reason, single-carrier or single-carrier-like modulation schemes like DFT-precoded OFDM are often used when there are stringent requirements on power efficiency of the RF amplifiers. Single-carrier signals have a much lower peak-toaverage ratio and can be shaped to have constant envelope even in multiuser MIMO systems [6].

The contributions made in this paper are summarized as follows. 1) We firstly propose a low complexity single carrier transmission scheme for the frequency selective MU-MISO GBC. 2) At low total transmit power to receiver noise ratio, the proposed transmission scheme is shown to effectively suppress intersymbol interference (ISI) and MU interference at each receiver, thereby achieving near optimal sum-rate performance. 3) Additionally, the proposed scheme does not require any receiver equalization. Also, its simplicity allows for separate, decentralized computation at each BS antenna. 4) An achievable information sum-rate is derived for the proposed scheme. This sum-rate is further shown to be invariant of the channel PDP. 5) In terms of power efficiency, the proposed scheme is shown to exhibit an array power gain proportional to the number of BS antennas.

\section{SySTEM MOdEL}

A frequency selective MU-MISO downlink channel is considered, with $M$ BS antennas and $K$ single antenna users. The channel between the $m$-th transmit antenna and the $k$ th user is modeled as a finite impulse response (FIR) filter with $L$ taps. The $l$-th channel tap is given by $\sqrt{d_{l}[k]} h_{l}^{*}[m, k]$, where $h_{l}^{*}[m, k]$ and $d_{l}[k]$ model the fast and slow varying components, respectively. In this paper we assume a model where $h_{l}^{*}[m, k]$ is fixed during the transmission of a block of $N$ symbols and varies independently from one block to another. However, the slowly varying component (i.e. $\left.\sqrt{d_{l}[k]}\right)$ is assumed to be fixed throughout the entire communication. We further assume $h_{l}^{*}[m, k]$ to be i.i.d. $\mathcal{C N}(0,1)$ distributed. $d_{l}[k] \geq 0, l=0, \ldots, L-1$ models the PDP of the frequency 
selective channel for the $k$-th user. ${ }^{1}$ Let $x_{m}[i]$ be the symbol transmitted from transmit antenna $m$ at time $i$. The received signal at user $k$ at time $i$ is then given by

$$
y_{k}[i]=\sum_{l=0}^{L-1} \sum_{m=1}^{M} \sqrt{d_{l}[k]} h_{l}^{*}[m, k] x_{m}[i-l]+n_{k}[i],
$$

where $n_{k}[i]$ is the $\mathcal{C N}(0,1)$ distributed AWGN at the $k$-th receiver at time $i$. Define $\boldsymbol{y}[i] \triangleq\left[y_{1}[i], \ldots, y_{K}[i]\right]^{T} \in \mathbb{C}^{K}$ to be the vector of received user symbols at time $i$. Similarly, let $\boldsymbol{x}[i] \triangleq\left[x_{1}[i], \ldots, x_{M}[i]\right]^{T} \in \mathbb{C}^{M}$ be the transmitted vector at time $i$. Let $\boldsymbol{n}[i] \triangleq\left[n_{1}[i], \ldots, n_{K}[i]\right]^{T}$, with independent components. The received signal vector at time $i$ is given by $\boldsymbol{y}[i]=\sum_{l=0}^{L-1} \boldsymbol{D}_{l}^{1 / 2} \boldsymbol{H}_{l}^{H} \boldsymbol{x}[i-l]+\boldsymbol{n}[i]$, where $\boldsymbol{D}_{l} \triangleq$ $\operatorname{diag}\left\{d_{l}[1], \ldots, d_{l}[K]\right\}$, and $\boldsymbol{H}_{l} \in \mathbb{C}^{M \times K}$ is a matrix whose $(m, k)$-th element is $h_{l}[m, k]$. Also the channel PDP for each user is normalized such that

$$
\sum_{l=0}^{L-1} d_{l}[k]=1, \forall k=1, \ldots, K .
$$

The BS is assumed to have full CSI, whereas the users have knowledge of the channel statistics only. ${ }^{2}$

Let $s_{k}[i]$ denote the information symbol to be communicated to the $k$-th user at time $i$. The information symbol vector $s[i]=\left[s_{1}[i], \ldots, s_{K}[i]\right]^{T}$ is considered to have i.i.d. $\mathcal{C N}(0,1)$ components, i.e. $\mathbb{E}\left[\boldsymbol{s}[i] \boldsymbol{s}^{H}[i+j]\right]=\boldsymbol{I}_{K} \delta_{j}$, $\mathbb{E}\left[s[i] s^{T}[i+j]\right]=\mathbf{0}$. In this paper we propose a precoding scheme, where the transmitted vector at time $i$ is given by

$$
\boldsymbol{x}[i]=\sqrt{\frac{\rho_{f}}{M K}} \sum_{l=0}^{L-1} \boldsymbol{H}_{l} \boldsymbol{D}_{l}^{1 / 2} \boldsymbol{s}[i+l],
$$

where $\rho_{f} \triangleq \mathbb{E}\left[\|\boldsymbol{x}[i]\|^{2}\right]$ is the long-term average total power radiated by the BS antennas. In the following, we derive an achievable sum-rate for the proposed precoder in (3).

\section{Achievable Sum-Rate}

The bounding technique of [7], [8] is used here to obtain an achievable rate. In the following, a set of achievable rates is presented. For notational brevity we define $\boldsymbol{v}_{l}[k] \triangleq$ $\boldsymbol{H}_{l} \boldsymbol{D}_{l}^{1 / 2} \boldsymbol{e}_{k}$, where $\boldsymbol{e}_{k}$ is the all-zero vector except for the $k$ th component which is equal to 1 . Using (1) and (3) the signal received by user $k$ at time $i$ is given by

$$
y_{k}[i]=\underbrace{\left(\sqrt{\frac{\rho_{f}}{M K}} \sum_{l=0}^{L-1} \mathbb{E}\left[\boldsymbol{v}_{l}^{H}[k] \boldsymbol{v}_{l}[k]\right]\right) s_{k}[i]}_{\text {Desired Signal Term }}+\underbrace{n_{k}^{\prime}[i],}_{\text {Effective Noise Term }}
$$

where ${ }^{3}$

$$
n_{k}^{\prime}[i] \triangleq \underbrace{\sqrt{\frac{\rho_{f}}{M K}}\left(\sum_{l=0}^{L-1} \boldsymbol{v}_{l}^{H}[k] \boldsymbol{v}_{l}[k]-\sum_{l=0}^{L-1} \mathbb{E}\left[\boldsymbol{v}_{l}^{H}[k] \boldsymbol{v}_{l}[k]\right]\right) s_{k}[i]}_{\text {Additional Interference Term (IF) }}
$$

${ }^{1}$ PDP determines the distribution of the received power across different channel taps.

${ }^{2}$ In a time division duplex (TDD) system, CSI at the BS can be acquired through uplink training and exploiting the uplink-downlink channel reciprocity.

${ }^{3}$ Following [7], [8], we have split the coefficient of the term $\sqrt{\frac{\rho_{f}}{M K}} \sum_{l=0}^{L-1} \boldsymbol{v}_{l}[k]^{H} \boldsymbol{v}_{l}[k] s_{k}[i]$ into a sum of its mean value (which is known to the receiver) and the deviation around its mean.

$$
\begin{aligned}
& +\underbrace{\sqrt{\frac{\rho_{f}}{M K}} \sum_{\substack{a=1-L \\
a \neq 0}}^{L-1} \sum_{\substack{l=\max (a, 0) \\
\text { Intersymbol Interference (ISI) }}}^{\min (L-1+a, L-1)} \boldsymbol{v}_{l}^{H}[k] \boldsymbol{v}_{l-a}[k] s_{k}[i-a]}_{\text {Multiuser Interference (MUI) }} \\
& +\underbrace{\sqrt{\frac{\rho_{f}}{M K}} \sum_{\substack{q=1 \\
q \neq k}}^{K} \sum_{\substack{a=1-L \\
L-1}}^{\min (L-1+a, L-1)} \sum_{\substack{l=\max (a, 0) \\
n_{k}[i]}}^{n_{l}^{H}[k] \boldsymbol{v}_{l-a}[q] s_{q}[i-a]}}_{\text {AWGN }}
\end{aligned}
$$

is the effective noise term. This term includes (i) the IF term which represents the variation of the desired signal around its mean, (ii) the ISI term between the current symbol of user $k$, i.e. $s_{k}[i]$, and the symbols intended to the same user at other time instances (i.e. $s_{k}[i+j], j \neq 0$ ), (iii) the MUI term due to the information symbols intended for other users and, (iv) the AWGN term. In the proposed precoder, each user's codeword is long enough such that it spans across multiple coherence intervals. With long codewords, the effective variance of $n_{k}^{\prime}[i]$ is no longer dependent on a particular channel realization but only depends on the channel statistics. From this it follows that the desired signal $s_{k}[i]$ is uncorrelated with the effective noise $n_{k}^{\prime}[i]$, i.e. $\mathbb{E}\left[s_{k}[i] n_{k}^{\prime}[i]\right]=0$, where the expectation is taken over the channel realizations, the information symbols and additive noise. Therefore, with long codewords the channel is effectively an additive noise channel with the noise $n_{k}^{\prime}[i]$ being non-Gaussian and uncorrelated to the information symbol $s_{k}[i]$. Further, the user has perfect knowledge of its channel statistic and therefore it knows the scaling factor $\sum_{l=0}^{L-1} \mathbb{E}\left[\boldsymbol{v}_{l}^{H}[k] \boldsymbol{v}_{l}[k]\right]$. Hence, an achievable information rate for the channel in (4) is given by considering the worst case uncorrelated additive noise having the same variance as $n_{k}^{\prime}[i]$. Given that the data signal $s[i]$ is Gaussian, the worst uncorrelated additive noise is circularly symmetric Gaussian distributed with the same variance as $n_{k}^{\prime}[i]$. Therefore, the following information rate is achievable for the $k$-th user

$$
R_{k}=\log _{2}\left(1+S_{k} / \operatorname{Var}\left(n_{k}^{\prime}[i]\right)\right)
$$

where $S_{k}=\mathbb{E}_{s_{k}[i]}\left[\left|\sqrt{\frac{\rho_{f}}{M K}} \sum_{l=0}^{L-1} \mathbb{E}\left[\boldsymbol{v}_{l}^{H}[k] \boldsymbol{v}_{l}[k]\right] s_{k}[i]\right|^{2}\right]$ is the average power of the desired signal term in (4) and $\operatorname{Var}\left(n_{k}^{\prime}[i]\right) \triangleq \mathbb{E}\left[\left|n_{k}^{\prime}[i]-\mathbb{E}\left[n_{k}^{\prime}[i]\right]\right|^{2}\right]$.

Proposition 1: The variance of $n_{k}^{\prime}[i]$ is invariant of any PDP that satisfies (2), and is given by

$$
\operatorname{Var}\left(n_{k}^{\prime}[i]\right)=\rho_{f}+1 .
$$

Proof: Using (5), the effective noise variance is given by

$$
\begin{aligned}
\operatorname{Var}\left(n_{k}^{\prime}[i]\right) & =\frac{\rho_{f}}{K} \sum_{q=1}^{K} \sum_{a=1}^{L-1} \sum_{l=a}^{L-1}\left(d_{l-a}[k] d_{l}[q]+d_{l}[k] d_{l-a}[q]\right) \\
& +\frac{\rho_{f}}{K} \sum_{q=1}^{K} \sum_{l=0}^{L-1} d_{l}[k] d_{l}[q]+1,
\end{aligned}
$$

where the expectation is taken over the statistics of $\boldsymbol{H}_{l}, l=$ $\{0, \ldots, L-1\}, \boldsymbol{s}[i+a], a=\{1-L, \ldots, L-1\}$ and $n_{k}[i]$. Define $\boldsymbol{\Delta} \in \mathbb{R}^{K \times L}$ such that $[\boldsymbol{\Delta}]_{i, j}=d_{j-1}[i]$ and let $\mathbf{1} \in$ 
$\{1\}^{L \times L}$ denote the matrix with all entries equal to one. Then, (8) can be expressed as

$$
\begin{aligned}
\operatorname{Var}\left(n_{k}^{\prime}[i]\right) & =\frac{\rho_{f}}{K} \sum_{q=1}^{K} \sum_{a=1}^{L-1} \sum_{l=a}^{L-1} \boldsymbol{e}_{k}^{T} \boldsymbol{\Delta}\left(\boldsymbol{e}_{l-a+1} \boldsymbol{e}_{l+1}^{T}\right. \\
& \left.+\boldsymbol{e}_{l+1} \boldsymbol{e}_{l-a+1}^{T}\right) \boldsymbol{\Delta}^{T} \boldsymbol{e}_{q}+\frac{\rho_{f}}{K} \sum_{q=1}^{K} \boldsymbol{e}_{k}^{T} \boldsymbol{\Delta} \boldsymbol{\Delta}^{T} \boldsymbol{e}_{q}+1 \\
& =\frac{\rho_{f}}{K} \sum_{q=1}^{K} \boldsymbol{e}_{k}^{T} \boldsymbol{\Delta} \mathbf{1} \boldsymbol{\Delta}^{T} \boldsymbol{e}_{q}+1 .
\end{aligned}
$$

From (2) it follows that $\boldsymbol{e}_{k}^{T} \boldsymbol{\Delta} \mathbf{1}=\left[\begin{array}{lll}1 & \ldots & 1\end{array}\right]$. Using this fact in (9) completes the proof.

It is apparent from (7) that the variance of the effective noise consists of the variance of the white noise term (which is 1) and the variance of the sum of interference terms (which is $\rho_{f}$ ). In the following we provide an explanation as to why the variance of the effective noise term is invariant of the PDP. Note that the precoder in (3) is like a matching pre-filter whose impulse response is a time reversed and complex-conjugated image of the channel impulse response (CIR). Due to this special structure of the proposed precoder, $n_{k}^{\prime}[i]$ is composed of terms which consist of all non-zero auto-correlation lags of the CIR for the $k$-th user (ISI term in (5)), as well as all crosscorrelation lags between the CIR of user $k$ and the CIR of the remaining $(K-1)$ users (MUI term in (5)). The effective MUI in $y_{k}[i]$ from the symbols intended for the $q$-th user, depends only upon the total power in all channel correlation lags between the CIR's of the $k$-th and the $q$-th user. Due to the same channel and information symbol statistics for all users, the effective MUI in $y_{k}[i]$ from each of the remaining $(K-1)$ users is identical, and is independent of the individual PDPs (the total power in the cross-correlation lags depends only upon the total power in the CIR for each user, which is independent of $k$ due to (2)).

Further, the useful signal term in $y_{k}[i]$ is proportional to the zero-lag auto-correlation of the CIR for the $k$ th user. This zero-lag auto-correlation (i.e. maximum gain combining of the lags) is proportional to the total channel power gain (combining all taps) from the $M$ BS antennas to the $k$-th user and is therefore $O(M)$. The average power of the desired signal term in (4) is given by $\mathbb{E}_{s_{k}[i]}\left[\left|\sqrt{\frac{\rho_{f}}{M K}} \sum_{l=0}^{L-1} \mathbb{E}\left[\boldsymbol{v}_{l}^{H}[k] \boldsymbol{v}_{l}[k]\right] s_{k}[i]\right|^{2}\right]=\rho_{f} M / K$. Using this fact and (7) in (6), the achievable rate $R_{k}$ for user $k$ is given by $R_{k}=\log _{2}\left(1+\rho_{f} M /\left(K \rho_{f}+K\right)\right)$. The achievable sum-rate is therefore given by

$$
R_{\text {sum }}\left(\rho_{f}, M, K\right)=\sum_{k=1}^{K} R_{k}=K \log _{2}\left(1+\frac{\rho_{f} M}{K \rho_{f}+K}\right) .
$$

For the sake of comparison, we also consider a co-operative upper bound on the sum-capacity of the frequency selective GBC. ${ }^{4}$ Essentially, we get an upper bound by considering the users to be co-operative, which reduces the MU channel to a single user MIMO channel, with perfect CSI at both the transmitter and the receiver. We further consider transmission

\footnotetext{
${ }^{4}$ The sum-capacity of the MIMO GBC is known. However, for the results reported in this paper, it suffices to consider only the co-operative upper bound on the sum-capacity.
}

in time with large blocks (block size $\gg L$ ), where in each block the last few transmit vectors are zeros so as to avoid any inter-block interference. The sum-capacity for this single user MIMO block channel is given by beamforming along the right singular vectors of the effective channel matrix, thus transforming the channel into a set of parallel channels. Gaussian symbols are communicated over the parallel channels and power allocation is given by the waterfilling scheme.

With i.i.d complex normal entries in $\boldsymbol{H}_{l}$, it is clear that for fixed $K, \frac{\boldsymbol{H}_{l}^{H} \boldsymbol{H}_{l}}{M} \rightarrow \boldsymbol{I}_{K}$ as $M \rightarrow \infty$. Therefore for $M \gg K$, the $K$ singular values of $\boldsymbol{H}_{l}$ are all roughly equal to $\sqrt{M}$ (i.e., the power gain for each parallel channel is $\approx M$ ). With a uniform power allocation of $\rho_{f} / K$ across parallel channels, the co-operative upper bound on the ergodic sum-capacity of the GBC is given by

$$
C_{\text {coop }}\left(\rho_{f}, M, K\right) \approx K \log _{2}\left(1+\rho_{f} M / K\right) .
$$

We conclude our analysis with two propositions on the nearoptimality and the array gain of the proposed precoder.

Proposition 2: When $\rho_{f} \ll 1$ and $M \gg K, R_{\text {sum }} \approx C_{\text {coop }}$ and the proposed precoder is near-optimal.

Proof: Observe that when $\rho_{f} \ll 1$, the effective noise variance, $\operatorname{Var}\left(n_{k}^{\prime}\right)=\rho_{f}+1 \approx 1$ (essentially the additive white noise dominates over the interference terms in (5)). It follows that, $K \rho_{f}+K \approx K$ and therefore the expressions in (10) and (11) are approximately equal.

Proposition 3: The proposed precoder exhibits an $O(M)$ array power gain.

Proof: For the proposed precoder, using (10) the minimum transmit power $\rho_{f}$ required to achieve a fixed desired sum-rate $R_{\text {sum }}$ with $K$ users and $M \mathrm{BS}$ antennas is given by $\rho_{f}(M)=\frac{K\left(2^{R_{\text {sum }} / K}-1\right)}{M+K\left(2^{R_{\text {sum }} / K}-1\right)}$. Since $\lim _{M \rightarrow \infty} \frac{1}{M} \frac{\rho_{f}(1)}{\rho_{f}(M)}=$ $\frac{1}{1+K\left(2^{R_{\mathrm{sum}} / K}-1\right)}>0$ from [9] it follows that the proposed precoder achieves an $O(M)$ array power gain.

This implies that for a sufficiently large $M, \rho_{f}(M) / \rho_{f}(1) \propto$ $1 / M$ (i.e. the total transmitted power can be reduced linearly by increasing the number of BS antennas). A similar analysis of the co-operative sum-capacity (see (11)) reveals that the array power gain achieved by a sum-capacity achieving scheme is also $O(M)$.

\section{Simulation Results}

In the following, representative simulation results are presented, where the performance of proposed precoder is compared to the co-operative upper bound. Throughout the conducted simulations, the PDP is exponential with $L=4$ and $d_{l}[k]=\frac{e^{-\theta_{k} l}}{\sum_{i=0}^{3} e^{-\theta_{k}}}, \quad l=\{0, \ldots, 3\}$, where $\theta_{k}=$ $(k-1) / 5, k=\{1, \ldots, K\}$. As proved in Proposition 1, the achievable sum-rate is invariant of the PDP. Hence, any other PDP which satisfies (2), would also yield the same results. Proposition 2 is supported by Fig. 1, where the sum-rate is plotted as a function of $\rho_{f}$, for $M=50$ and $K=10$. The sum-rate performance of the proposed precoder is given both by the theoretical expression (10) and via simulations. Similarly, the co-operative sum-capacity upper bound is calculated via simulations and by the approximation in (11). For 


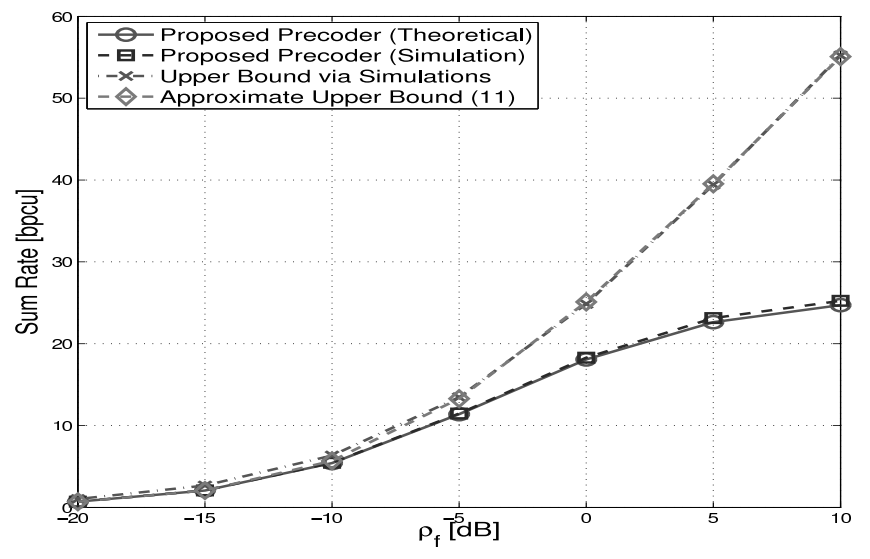

Fig. 1. Sum rate of the proposed precoder and the co-operative sum-capacity upper bound vs $\rho_{f}$, calculated for $K=10$ users, $M=50 \mathrm{BS}$ antennas.

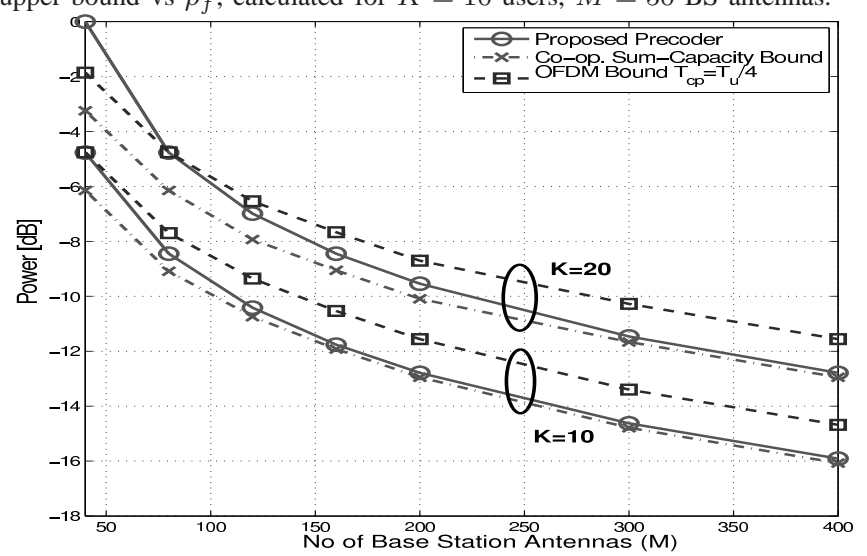

Fig. 2. Minimum required transmit power to achieve a fixed per user information rate $r=1 \mathrm{bpcu}$ as a function of the number of BS antennas.

$\rho_{f} \ll 1(0 \mathrm{~dB})$, as can be seen in Fig. 1, the performance of the proposed precoder is similar to the upper bound, implying optimality. Note that as $\rho_{f}$ increases, the interference terms dominate over the white noise term in (5) and the effective noise variance is therefore $\rho_{f}+1 \approx \rho_{f}$. Hence, as $\rho_{f} \rightarrow \infty$ the sum-rate of the proposed precoder saturates to the value $K \log _{2}(1+M / K)=25.85$ bpcu. It can also be seen that the approximation to the sum-capacity upper bound is tight.

The analytical result in Proposition 3 is supported by Fig. 2, where for a fixed number of users and a fixed per user rate of 1 bpcu, the minimum total transmit power required is plotted as a function of the number of BS antennas. In Fig. 2 it is observed that the minimum transmit power required by the proposed precoder can be reduced by roughly $3 \mathrm{~dB}$ with every doubling in the number of the BS antennas (for sufficiently large $M$ ). This implies the achievability of an $O(M)$ array power gain, as stated in Proposition 3. In Fig. 2 it is also observed that for sufficiently large values of $M$ the total transmit power required by the proposed precoder is roughly equal to the total transmit power required by a sum-capacity achieving scheme. Further, for the sake of comparison, consider a typical scenario, where OFDM is used. Let $\rho_{f}^{\text {OFDM }}$ denote the total transmit power for OFDM transmission. Under OFDM transmission with $M \gg$ $K$, it can be shown that the per user ergodic information rate (in i.i.d. Rayleigh fading channel) is given by

$$
r \approx \frac{T_{u}}{T_{u}+T_{\mathrm{cp}}} \log _{2}\left(1+\rho_{f}^{\mathrm{OFDM}} \frac{M}{K}\right),
$$

where $T_{\mathrm{cp}}$ is the duration of the cyclic prefix and $T_{u}$ is the duration of the useful signal. ${ }^{5}$ From (12) it follows that, to achieve an ergodic per user information rate of $r$ bpcu the minimum required total transmit power is given by $\rho_{f}^{\mathrm{OFDM}}(r) \approx$ $\frac{K}{M}\left(2^{r\left(T_{u}+T_{\mathrm{cp}}\right) / T_{u}}-1\right)$. For a given desired per user ergodic information rate, the additional total transmit power required under OFDM transmission when compared to an optimal GBC sum-capacity achieving scheme is upper bounded by $\rho_{f}^{\text {OFDM }}(r) / \rho_{f}^{\text {coop }}(r)$, where $\rho_{f}^{\text {coop }}(r)=\left(2^{r}-1\right) K / M$ is roughly equal to the required transmit power for the co-operative sumcapacity bound to be $r K$ bpcu (see (11)). The additional transmit power required under OFDM transmission is therefore given by $\frac{2^{r\left(1+T_{\mathrm{cp}} / T_{u}\right)}-1}{2^{r}-1}$. Since $\frac{2^{r\left(1+T_{\mathrm{cp}} / T_{u}\right)}-1}{2^{r}-1}>1$ and the total transmit power required by the proposed precoder is roughly equal to that required by a sum-capacity achieving scheme $\left(M \gg K\right.$ and $\rho_{f} \ll 1$ (see Proposition 2)), it can be concluded that the proposed precoder is more power efficient than OFDM transmission for large $M / K$. As an example, for a typical IEEE 802.11a scenario with $T_{\mathrm{cp}}=T_{u} / 4$, a desired per user information rate $r=1 \mathrm{bpcu}$ and $M \gg K$, this additional transmit power required under OFDM transmission when compared to the proposed precoder is $1.39 \mathrm{~dB}$. The minimum transmit power required under OFDM transmission is also plotted in Fig. 2, where it can be seen that for $M>4 K$ the proposed precoding scheme is more power efficient than OFDM transmission and requires no equalization at the receiver (Note that equalization in OFDM receivers requires FFT processing).

\section{REFERENCES}

[1] G. Foschini and M. Gans, "On limits of wireless communications in a fading environment when using multiple antennas," Wireless Pers. Comm., vol. 6, pp. 311-335, Mar. 1998.

[2] D. Gesbert and et al., "Shifting the MIMO paradigm," IEEE Sig. Proc. Mag., vol. 24, pp. 36-46, Sep. 2007.

[3] F. Rusek, D. Persson, B. K. Lau, E. G. Larsson, T. L. Marzetta, O. Edfors, and F. Tufvesson, "Scaling up MIMO: Opportunities and challenges with very large arrays," IEEE Sig. Proc. Mag., to appear, vol. arXiv:1201.3210v1, 2012.

[4] T. L. Marzetta, "Noncooperative cellular wireless with unlimited numbers of base station antennas," IEEE Trans. Wireless Comm., vol. 9, pp. 35903600, Nov. 2010.

[5] H. Q. Ngo, E. G. Larsson, and T. L. Marzetta, "Energy and spectral efficiency of very large multiuser MIMO systems," submitted to the IEEE Trans. Comm., vol. arXiv:1112.3810, 2011.

[6] S. K. Mohammed and E. G. Larsson, "Per-antenna constant envelope precoding for large multi-user MIMO systems," submitted to the IEEE Trans. Comm., vol. arXiv:1201.1634v1.

[7] B. Hassibi and B. M. Hochwald, "How much training is needed in multiple-antenna wireless links?," IEEE Trans. Inf. Theory, vol. 49, pp. 951-963, Apr. 2003.

[8] T. L. Marzetta, "How much training is required for multiuser MIMO?," in Proc. 40th Asilomar Conf. ACSSC '06, pp. 359 -363, Nov. 2006.

[9] D. Tse and P. Viswanath, Fundamentals of Wireless Communications. Cambridge, UK: Cambridge Univ. Press, 2004.

${ }^{5}$ Note that in practice, modern wireless standards employ $X>1$ OFDM symbols per coherence time interval. Each OFDM symbol consists of $N_{u}$ channel uses for data transmission and $N_{\mathrm{cp}}$ channel uses for the cyclic prefix. This means that $X N_{\text {cp }}$ channel uses in each coherence time interval are used for non-data transmission. In contrast, in the proposed precoding scheme only $N_{\mathrm{cp}}$ channel uses per coherence time interval are used for nondata transmission. (These $N_{\mathrm{cp}}$ channel uses are used for zero-padding at the beginning of each coherence interval.) Note that in practical wireless standards $X \gtrsim 10$, which implies that the proposed precoder makes better use of available channel bandwidth. 